\title{
Quercetin promotes the apoptosis of fibroblast-like synoviocytes in rheumatoid arthritis by upregulating IncRNA MALAT1
}

\author{
FANG PAN $^{1}$, LIHUA ZHU ${ }^{1}$, HAOZHE LV ${ }^{1}$ and CHUNPENG PEI ${ }^{2}$ \\ ${ }^{1}$ Department of Rheumatology, Daqing Oilfield General Hospital, Daqing; ${ }^{2}$ Department of Renal Diseases, \\ The First Affiliated Hospital, Heilongjiang University of Chinese Medicine, Harbin, Heilongjiang, P.R. China
}

Received August 6, 2015; Accepted March 8, 2016

DOI: $10.3892 /$ ijmm.2016.2755

\begin{abstract}
Rheumatoid arthritis (RA) is a chronic autoimmune joint disease and fibroblast-like synoviocytes (FLS) are the resident mesenchymal cells of synovial joints. Quercetin is a dietary antioxidant. In this study, we aimed to explore the mechanisms responsible for the quercetin-induced apoptosis of FLS from patients with RA (termed RAFLS). RAFLS viability was determined following treatent of the cells with or without quercetin using the Cell Counting kit-8 (CCK-8) assay. The apoptosis of the RAFLS was analyzed using the Annexin V-fluorescein isothiocyanate (FITC) apoptosis detection kit I. The results revealed that RAFLS viability decreased and apoptosis increased in following treatment with quercetin. The differentially expressed long non-coding RNAs (IncRNAs) were screened and marked by PCR array following treatment with quercetin. The expression levels of the screened lncRNAs were then determined and compared in the cells treated with or without quercetin by quantitative PCR. The lncRNA metastasis associated lung adenocarcinoma transcript 1 (MALAT1) was finally selected. Small interfering RNA (siRNA) was then used to knock down the expression of MALAT1 in order to determine the role of MALAT1 in the quercetin-induced apoptosis of RAFLS. The results revealed that the knockdown of MALAT1 inhibited RAFLS apoptosis. At the same time, the expression of caspase- 3 and caspase- 9 was significantly decreased in the cells in which MALAT1 was knocked down. The phosphoinositide 3-kinase (PI3K)/AKT signaling pathway was activated; this activation is known to be associated with enhanced cell proliferation and decreased apoptosis. The findings of our study indicate that quercetin promotes RAFLS apoptosis by upregulating lncRNA MALAT1, and that MALAT1 induces apoptosis by inhibiting the activation of the PI3K/AKT pathway.
\end{abstract}

Correspondence to: Dr Chunpeng Pei, Department of Renal Diseases, The First Affiliated Hospital, Heilongjiang University of Chinese Medicine, 26 Heping Road, Dongli, Harbin, Heilongjiang 150040 , P.R. China

E-mail: chppei@yeah.net

Key words: rheumatoid arthritis fibroblast-like synoviocytes, MALAT1, caspase, PI3K/AKT, apoptosis

\section{Introduction}

Rheumatoid arthritis (RA) is a chronic autoimmune joint disease and is characterized by the proliferation of synoviocytes that produce inflammatory cytokines and chemokines (1). Despite significant therapeutic advances, there is still a need for effective treatments for RA (2). RA not only decreases the quality of life and life expectancy of patients, but also and most commonly, accelerates atherosclerosis (3). The hallmarks of RA are leukocytic infiltration of the synovium and the expansion of fibroblast-like synoviocytes (FLS) (4). FLS are the resident mesenchymal cells of synovial joints and are located in the lining of the joints. They play an increasingly important role in the pathogenesis of RA, and participate in all the pathological events of RA (5).

Apoptosis is a key mechanism that regulates tissue composition and homeostasis (6). It can be considered a therapeutic tool in RA, and alterations in the apoptosis of synovial cells have been described in residential synoviocytes, as well as in inflammatory cells $(7,8)$. Long non-coding RNAs (lncRNAs) are a recently discovered class of non-protein coding RNAs $(9,10)$, and metastasis associated lung adenocarcinoma transcript 1 (MALAT1), a well-described lncRNA of of $>8,000 \mathrm{nt}$ in length expressed on chromosome 11q13, is widely expressed in normal tissues (11-13). MALAT1 has been recently observed to be upregulated in various human cancers and has been shown to be associated with cancer metastasis and recurrence of hepatocellular carcinoma following liver transplantation (14). In bladder cancer, the upregulation of MALAT1 has been reported to contribute to enhanced cell migration by inducing epithelial-to-mesenchymal transition (15). MALAT1 has also been reported to control cell cycle progression by regulating the expression of the oncogenic transcription factor, B-MYB (16). In osteosarcoma, MALAT1 has been shown to promote the proliferation and metastasis of osteosarcoma cells by activating the phosphoinositide 3-kinase (PI3K)/AKT pathway (17). The P13K/AKT signaling pathway plays a critical role in regulating basic cellular functions, such as the control of transcription and translation (18). The P13K/AKT pathway is also involved in apoptosis; when this pathway is activated, apoptosis is then decreased and cell proliferation increases (19).

Quercetin is a flavonoid molecule ubiquitous in nature (20). It is a dietary antioxidant that prevents the oxidation of lowdensity lipoprotein in vitro by the scavenging of free oxygen radicals and the inhibition of the growth of certain types of cancer cell $(21,22)$. It has been shown to prevent and protect 
against streptozotocin-induced oxidative stress and $\beta$-cell damage in the rat pancreas (23). Quercetin has been shown to be effective in the management of arthritis (24). It has also been shown to inhibit the release of macrophage-derived cytokines and nitric oxide (25).

In this study, we aimed to elucidate the mechanisms through which quercetin affects FLS from patients with RA (termed RAFLS) and the involvement of possible signaling pathways. First, we determined the relative expression levels of differentially expressed lncRNAs and the proliferation of RAFLS, and screened the most significantly differentially expressed lncRNA. The screened lncRNA was then knocked down and the apoptosis of RAFLS was analyzed. At the same time, the protein expression levels of caspase-3 and caspase-9, Bax, Bcl-2, phosphorylated (p-)P13K, P13K, p-AKT, AKT, p-mammalian target of rapamycin (mTOR) and mTOR were determined by western blot analysis.

\section{Materials and methods}

Cell culture and treatment. Rheumatoid arthritis fibroblast-like synoviocytes (RAFLS) used in this study were obtained from the Shanghai Institute of Biochemistry and Cell Biology (Shanghai, China). The cells were cultured in Dulbecco's modified Eagle's medium (DMEM) containing 10\% fetal bovine serum (FBS), $100 \mathrm{U} / \mathrm{ml}$ penicillin and $100 \mu \mathrm{g} / \mathrm{ml}$ streptomycin in a humidified cell incubator at $37^{\circ} \mathrm{C}$ in a humidified atmosphere of $5 \% \mathrm{CO}_{2}$ and $95 \%$ air. The medium was changed every $24 \mathrm{~h}$ and cells at passages 3 to 8 were used in the following experiments. Each experiment performed thrice. All the samples were stored at $-80^{\circ} \mathrm{C}$ for further use.

Cell tranfection. MALAT1 and HOTAIR small interfering RNAs (siRNAs) and scrambled RNA were purchased from Dharmacon Research, Inc. (Lafayette, CO, USA). Transfection was conducted with cationic lipopolyamines (Invitrogen, Carlsbad, CA, USA). RAFLS at approximately 70-80\% confluence were used for transfection. siRNA targeting MALAT1 (siRNA-MALAT1-1, -2, -3 or si-MALAT1-1, $-2,-3)$, siRNA targeting HOTAIR or scrambled RNA control (siRNA-Scramble or si-Scramble) in Opti-MEM (Invitrogen) was added at a final concentration of $5 \mu \mathrm{g} / \mathrm{ml}$ DNA. The control cells represent normal RAFLS without any transfection. Following incubation for $4 \mathrm{~h}$, fresh growth medium was added, and the cells were cultured as described above.

RNA extraction and PCR array. Total RNA was extracted and isolated from the cells using the Agilent Technologies Total RNA isolation mini kit (Agilent Technologies, Palo Alto, CA, USA) according to the manufacturer's instructions. Spectrophotometric methods were used to assess the quality and quantity of the RNA samples. PCR array was used to screen the differentially expressed lncRNAs following treatment with quercetin.

A PCR array is a highthroughput RT-PCR system which is based on ATAC-PCR. A 96-well PCR plate was prepared with a different primer-probe in each well as the PCR array. In brief, the experimental procedure was as follows: RNA was converted into cDNA, digested by a restriction enzyme, and then ligated to an adaptor with the cohesive ends created by the enzyme. After mixing the ligated samples, PCR amplifica- tion was conducted with an adaptor primer and a gene-specific primer. The PCR specific procedure was: denaturing at $95^{\circ} \mathrm{C}$ for $15 \mathrm{sec}$, annealing for $30 \mathrm{sec}$ at $60^{\circ} \mathrm{C}$, and extension for $30 \mathrm{sec}$ at $72^{\circ} \mathrm{C}$. A total of 40 PCR cycles were performed.

Quantitative PCR ( $q P C R$ ). To quantitatively determine the messenger RNA (mRNA) expression levels of MALAT1, HOX transcript antisense RNA (HOTAIR), prostate cancer associated rranscript 1 (PCAT1), WD repeat containing, antisense to TP53 (WRAP53), long stress-induced non-coding transcript 5 (LSINCT5), 7SK, C1QTNF9B antisense RNA 1 (C1QTNF9B-AS1), breast cancer anti-estrogen resistance 4 (BCAR4), maternally expressed 3 (MEG3), carbonyl reductase 3 antisense RNA (CBR3-AS), growth arrest-specific 5 (GAS5), ZNFX1 antisense RNA 1 (ZNFX1-AS1), HIF1A antisense RNA 1 (HIF1A-AS1), DNM3 opposite strand/antisense RNA (DNM3OS), brain cytoplasmic RNA 1 (BCYRN1) and Yiya in the RAFLS treated with quercetin (RAFLS + quercetin) or in the untreated RAFLS (RAFLS - quercetin), as well as those of MALAT1 in normal cells (controls) or in the cells tranfected with siRNA-MALAT1 (si-MALAT1-1, -2, -3) or siRNA-Scramble (si-Scramble), qPCR was used. Genes were amplified by specific oligonucleotide primers, and the human glyceraldehyde 3-phosphate dehydrogenase (GAPDH) gene was used as an endogenous control. The primers were the following: PCAT-1 forward, 5'-AATGGCATGAACCTGGGAGGCG-3' and reverse, 5'-GGCTTTGGGAAGTGCTTTGGAG-3'; WRAP53 forward, 5'-TGGCACAAAGCTGGACAGT-3' and reverse 5'-GCTGGGTCCTGGTCTGAAG-3'; CBR3-AS1 forward, 5'-CTTCTGGTTACAATGATTCTC-3' and reverse, 5'-CACTTACTGCCTACATTAGA-3'; HIF1A-AS1 forward, 5'-AATGTGTTCCTTGCTCTT-3' and reverse, 5'-GTATGTCT CAGTTATCTTCCT-3'; LSINCT5 forward, 5'-TTCGGCAAG CTCCTTTTCTA-3' and reverse, 5'-GCCCAAGTCCCAAAA AGTTCT-3'; HOTAIR forward, 5'-TGCTACTTGTGTAGAC CCAG-3' and reverse, 5'-AGCAAAGGCTGGACCTTTGCT-3'; MALAT1 forward, 5'-TGATAGCCAAATTGAGACAA-3' and reverse 5'-TTCAGGGTGAGGAAGTAAAA-3'; 7SK forward, 5'-AAACAAGCTCTCAAGGTC-3' and reverse, 5'-CCTCATT TGGATGTGTCT-3'; BCAR4 forward, 5'-GGACTCATTGTT GTTCTAC-3' and reverse, 5'-ACCTATGGCTATCATTGTT-3'; GAS5 forward, 5'-CCCAAGGAAGGATGAG-3' and reverse, 5'-ACCAGGAGCAGAACCA-3'; MEG3 forward, 5'-CTGCCC ATCTACACCTCACG-3' and reverse, 5'-CTCTCCGCCGTCT GCGCTAGGGGCT-3'; CIQTNF9B -AS1 forward, 5'-CGGCG TGGTGTAGCGT-TC-3' and reverse, 5'-GTGCAGCCTGCGA CGGT-3'; Yiya forward, 5'-TATCCTATTCTTAGCAACTG-3' and reverse, 5'-ACATACCTGGCATATAGT-3'; ZNFX1-AS1 forward, 5'-CCAGTTCCACAAGGTTAC-3' and reverse, 5'-GC AGGTAGGCAGTTAGAA-3'; DNM3OS forward, 5'-ATAGA GCAAGTCTGGATT-3' and reverse, 5'-GGATGAGGCAATA ACATT-3'; BCYRN1 forward, 5'-CTGGGCAATATAGCGA GAC-3' and reverse, 5'-TGCTTTGAGGGAAGTTACG-3'; GAPDH forward, 5'-GAGTCAACGGATTTGGTCGT-3' and reverse, 5'-TTGATTTTGGAGGGATCTC-3'. The detection and quantification of mRNA expression involved the following steps: first, reverse transcription was performed at $55^{\circ} \mathrm{C}$ for $30 \mathrm{~min}$, initial activation for $15 \mathrm{~min}$ at $95^{\circ} \mathrm{C}$, next 40 cycles of denaturation were conducted at $94^{\circ} \mathrm{C}$ for $15 \mathrm{sec}$, then annealing for $30 \mathrm{sec}$ at $55^{\circ} \mathrm{C}$, extension for $30 \mathrm{sec}$ at $72^{\circ} \mathrm{C}$. The expression 

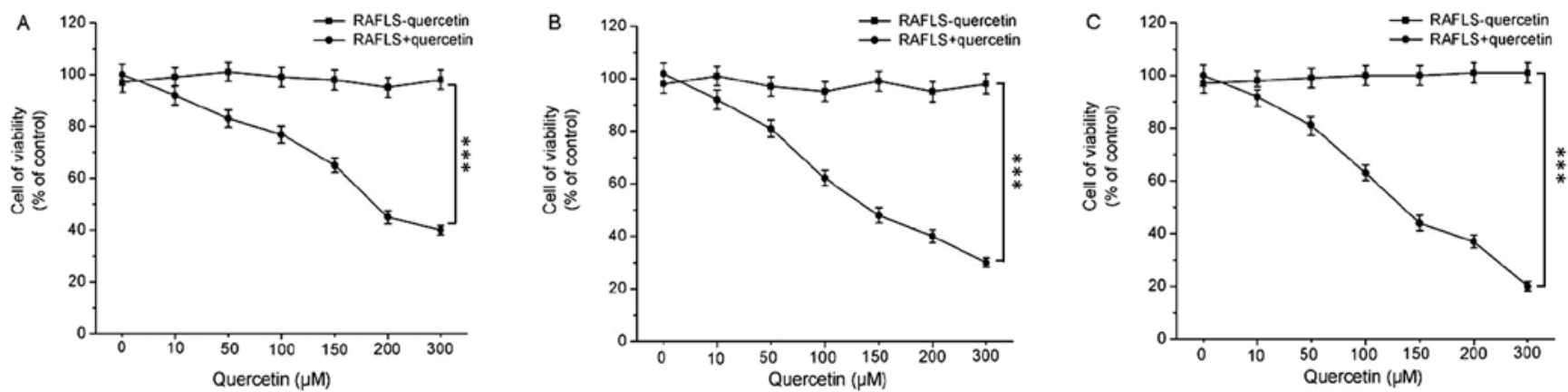

Figure 1. Effects of quercetin on the viability of RAFLS cultured in RPMI-1640 medium with $10 \%$ FBS and treated with various concentrations of quercetin for (A) $24 \mathrm{~h}$, (B) $48 \mathrm{~h}$ and (C) $72 \mathrm{~h}$. Each point is the mean \pm SD of 3 experiments. ${ }^{* * *} \mathrm{p}<0.001$ indicates significant difference compared to the controls.

level was normalized using U6 small nuclear RNA by the $2^{-\Delta \mathrm{Ct}}$ method. The $\triangle \mathrm{Ct}$ values were normalized to GAPDH level.

Western blot analysis. To determine the protein expression of signaling pathways which may be part of the molecular mechanisms involved in the effects of quercetin on RAFLS, western blot analysis was used. The cultured cells were lysed in RIPA buffer consisting of $1 \%$ sodium dodecyl sulfate (SDS), $0.1 \mathrm{mM}$ phenylmethylsulfonyl fluoride (PMSF) and complete protease inhibitors (Roche, Mannheim, Germany). The lysates were centrifuged at $13,000 \mathrm{x} \mathrm{g}$ for $15 \mathrm{~min}$, and the supernatants were frozen at $-80^{\circ} \mathrm{C}$ until use. The BCA protein assay kit (Pierce Chemical Co., Rockford, IL, USA) was used to measure the protein concentration of the lysates. Protein samples $(20 \mu \mathrm{g})$ prepared as indicated above were resolved by SDS-polyacrylamide gel electrophoresis and transferred onto nitrocellulose membranes (Millipore Corp., Bedford, MA, USA). After blocking in 5\% non-fat milk in Tris-buffered saline for $1 \mathrm{~h}$ at room temperature, the membranes were incubated in primary antibodies [including rabbit polyclonal to caspase-3 (1:500, Cat. no. ab13847; Abcam, Cambridge, MA, USA), mouse monoclonal to caspase-9 (1:300, Cat. no. sc-56076; Santa Cruz Biotechnology, Inc., Santa Cruz, CA, USA), rabbit polyclonal to Bax (1:500, Cat. no. ab10813; Abcam), rabbit polyclonal to $\mathrm{Bcl} 2$ (1:1,000,Cat.no.ab59348; Abcam), mouse monoclonal to GAPDH (1:400, Cat. no. sc-365062; Santa Cruz Biotechnology, Inc.), rabbit polyclonal to p-PI3K (1:300, Cat. no. BS4811; Biogot Technology Co., Ltd., Nanjing, China), rabbit polyclonal to PI3K (1:1,000, Cat. no. ab10813; Abcam), mouse monoclonal to Akt (1:200, Cat. no. sc-5298; Santa Cruz Biotechnology, Inc.), mouse monoclonal to p-Akt (1:800, Cat. no. ab38449; Abcam), rabbit polyclonal to mTOR $(1: 2,000$, Cat. no. ab2732; Abcam) and rabbit polyclonal to p-mTOR (1:800, Cat. no. ab1093; Abcam)], followed by horseradish peroxidase-conjugated secondary antibody (both from Santa Cruz Biotechnology, Inc.). The blots were scanned on the Fluor-S MAX MultiImager, and signal intensities were determined using Quantity One image software (both from Bio-Rad Laboratories, Inc., Hercules, USA).

Cell viability assay. To analyze RAFLS viability, the Cell Counting kit-8 (CCK-8) (Dojindo Laboratories, Kumamoto, Japan) was used in accordance with the manufacturer's instructions. An RAFLS suspension of $100 \mathrm{ml}$ following treatment with quercetin was plated in a 96-well plate supplemented with DMEM supplemented with $10 \%$ FBS with various concen- trations $(0,10,50,100,150,200$ and $300 \mu \mathrm{M})$ of quercetin (Sigma-Aldrich, St. Louis, MO, USA). Following culture for 24, 48 and $72 \mathrm{~h}$ at $37^{\circ} \mathrm{C}$ with $5 \% \mathrm{CO}_{2}, \mathrm{CCK}-8(10 \mathrm{ml})$ was added to each well. Following incubation for $1-4 \mathrm{~h}$ at $37^{\circ} \mathrm{C}$ with $5 \% \mathrm{CO}_{2}$, the optical density (OD) values were measured using an enzyme-labeled instrument (Varian Medical Systems, Inc., Palo Alto, CA, USA) at $450 \mathrm{~nm}$.

Analysis of apoptosis. RAFLS apoptosis in the control, si-MALAT1 and si-Scramble group was analyzed using an Annexin V-fluorescein isothiocyanate (FITC) Apoptosis Detection kit I (BD Pharmingen, Heidelberg, Germany) according to the manufacturer's instructions. Following transfection with si-MALAT1 or si-Scramble, the cells were collected by trypsinization and washed with phosphatebuffered saline (PBS), and then plated on 6-well plates at the concentration of $1 \times 10^{6}$ cells $/ \mathrm{ml}$. Following incubation for $72 \mathrm{~h}$ at $37^{\circ} \mathrm{C}$ with $5 \% \mathrm{CO}_{2}$, the cells were fixed by $70 \%$ pre-cooled ethanol and $100 \mu \mathrm{l}$ RNase $(10 \mathrm{mg} / \mathrm{ml})$ was then added. The cells were then stained with $5 \mu \mathrm{l}$ Annexin V-FITC and propidium iodide (PI). The samples were then incubated for $15 \mathrm{~min}$ in the dark. Apoptosis was analyzed using a FACSCalibur flow cytometry with CellQuest software (both from Becton-Dickinson, Mountain View, CA, USA). The cells undergoing apoptosis were Annexin V-FITC-positive and PI-negative.

Statistical analysis. The differences between the quercetin-treated and control cells were analyzed using the Student's t-test and a probability value of $\mathrm{p}<0.05$ was considered to indicate a statistically significant difference. All statistical analyses were performed using the SAS 6.12 software package.

\section{Results}

Cell viability following treatment with quercetin. In order to examine the effects of quercetin on RAFLS viability, the cells were treated with various concentrations of quercetin for 24,48 and $72 \mathrm{~h}$ and the RAFLS were then examined by flow cytometry. The results of cell viability determined at 24 , 48 and $72 \mathrm{~h}$ are shown in Fig. 1A-C, respectively. RAFLS viability markedly decreased following treatment with increasing concentrations of quercetin, and the cell viability also markedly decreased in a time-dependent manner even at the same quercetin concentration. The cell viability remained stable at all time points examined when no quercetin was used. 
A

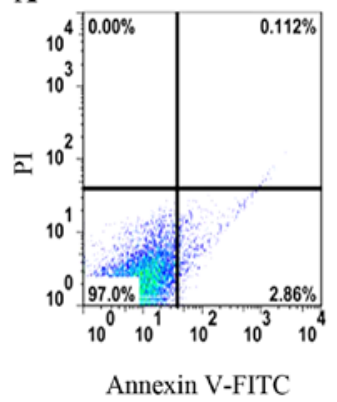

B

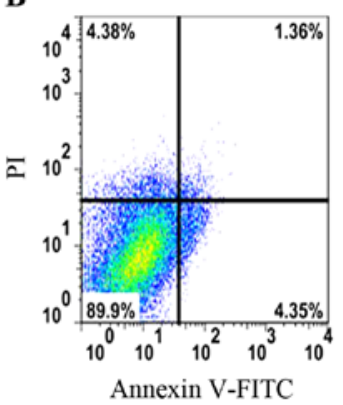

C

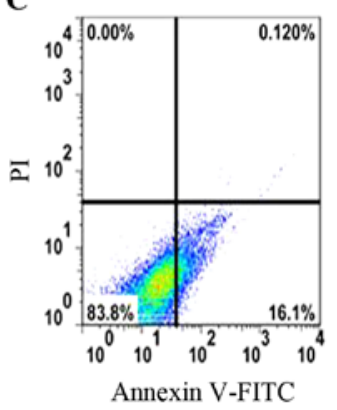

Figure 2. Effect of quercetin on the apoptosis of RAFLS treated with $200 \mu \mathrm{M}$ quercetin for (A) $24 \mathrm{~h}$, (B) $48 \mathrm{~h}$ and (C) $72 \mathrm{~h}$. Cells were stained with propidium iodide (PI) and analyzed by flow cytometry at $48 \mathrm{~h}$ post-transfection. Cells staining positive for Annexin V-fluorescein isothiocyanate (FITC) and negative for PI at 48 h post-transfection were considered to have undergone apoptosis. Upper-left area, dead cells; upper-right area, late-stage apoptotic cells; lower-left area, normal negative cells; lower-right area, early-stage apoptosis cells.

Cell apoptosis following treatment with quercetin. Apoptosis plays a key role in tissue homoeostasis both under physiological and pathological conditions (26). It has been demonstrated that certain characteristic changes occur in the composition and structure of the inflamed synovial membrane in RA (27). Therefore, in this study, the RAFLS apoptotic rate in the controls, or in the cells treated with $200 \mu \mathrm{M}$ quercetin (effective concentration from Fig. 1) for 24, 48 and $72 \mathrm{~h}$ was analyzed and the results are shown in Fig. 2. The apoptotic rate of the cells treated with quercetin for 24,48 and $72 \mathrm{~h}$ was $0.112,1.36$ and $0.120 \%$, respectively. These rates were much higher than those of the controls.

Treatment with quercetin results in the upregulation of lncRNA MALAT1. To explore the molecular mechanisms responsible for the effects of quercetin on RAFLS, a PCR array was used to analyze the changes in IncRNA expression. The relative expression levels of lncRNAs which were differentially expressed in the control and the group treated with quercetin are shown in Fig. 3A. The lncRNAs examined included PCAT1, CBR3-AS1, WRAP53, LSINCT5, HOTAIR, MALAT1, 7SK, C1QTNF9B-AS1, BCAR4, GAS5, MEG3, Yiya, ZNFX1-AS1, HIF1A-AS1, DNM3OS and BCYRN1; these lncRNAs all exhibited a difference in expression of $\geq 2$-fold, and those with the greatest differences in expression were marked. From these lncRNAs listed, the most significantly differentially expressed ones were selected: MALAT1, HOTAIR, MEG3, CBR3-AS1, GAS5 and Yiya. To further analyze the differences in the expression of these lncRNAs, their relative expression levels in the control cells and in the cells treated with quercetin were determined by qPCR and comparisons were made between the 2 groups of cells. As shown in Fig. 3B, MALAT1, HOTAIR and MEG3 were upregulated, whereas CBR3-AS1, GAS5 and Yiya were downregulated in cells treated with quercetin. Moreover, MALAT1 was upregulated by approximately 4-fold; therefore, MALAT1 was selected as the key lncRNA for analysis in the following experiments.

MALAT1 is necessary for the quercetin-induced apoptosis of RAFLS. To further determine whether the upregulation of MALAT1 is necessary for the quercetin-induced apoptosis of RAFLS, siRNAs were used to knock down the expression of MALAT1 and the results are shown in Fig. 4. The relative expression of MALAT1 in the RAFLS following transfection with si-MALAT1-1,-2, -3 was significantly decreased compared with the cells transfected with si-Scramble. Following transfection, western blot analysis was used to detect the expression level of MALAT1 in different groups. Transfection with si-MALAT1-2 induced the most significant decrease in MALAT1 expression (Fig. 4). Thus, si-MALAT1-2 was selected for use in the subsequent experiments.

We also examined the apoptosis of the control cells, and those transfected with si-MALAT1 or si-Scramble and the results are shown in Fig. 5. The apototic rate of the control cells, and those transfected with si-MALAT1 or si-Scramble was $0.159,0.120$ and $0.143 \%$, respectively. This indicated the that the knockdown of MALAT1 inhibited the apoptosis of the RAFLS.

Knockdown of MALAT1 influences protein expression. To explore the mechanisms responsible for the apoptosis induced by quercetin, we examined the involvement of caspase- 3 and caspase- 9 by measuring their expression levels in the cells in which MALAT1 was knocked down by western blot analysis. Bcl-2 family members have either pro-(i.e., Bax) or anti-apoptotic (i.e., Bcl-2) activities. The proteins of the Bcl-2 family regulate the mitochondrial pathway by controlling the permeabilization of the outer mitochondrial membrane in response to many types of stress or damage (28). Therefore, in this study, the expression of Bcl-2 and Bax was also determined. As shown in Fig. 6A, the protein expression of caspase-3 and caspase-9, as well as that of Bax in the cells transfected with si-MALAT1 was significantly lower than that of the cells transfected with si-Scramble. The protein expression of Bcl-2 increased in the cells transfected with si-MALAT1 compared with the cells transfected with the blank or si-Scramble.

Activation of the PI3K/AKT pathway by MALAT1 knockdown. The PI3K/AKT pathway is often aberrantly activated in human cancers and contributes to enhanced cell proliferation and metastasis $(29,30)$. In this study, to whether MALAT1 regulates the proliferation of RAFLS, western blot analysis was used to examine the effects of MALAT1 knockdown on two relative signaling pathways. The expression levels of proteins associated with the PI3K/AKT pathway were determined. As shown in Fig. 6B, the downregulation of MALAT1 significantly increased the levels of p-P13K, p-AKT and p-mTOR, and decreased those of AKT; no detectable changes were observed 


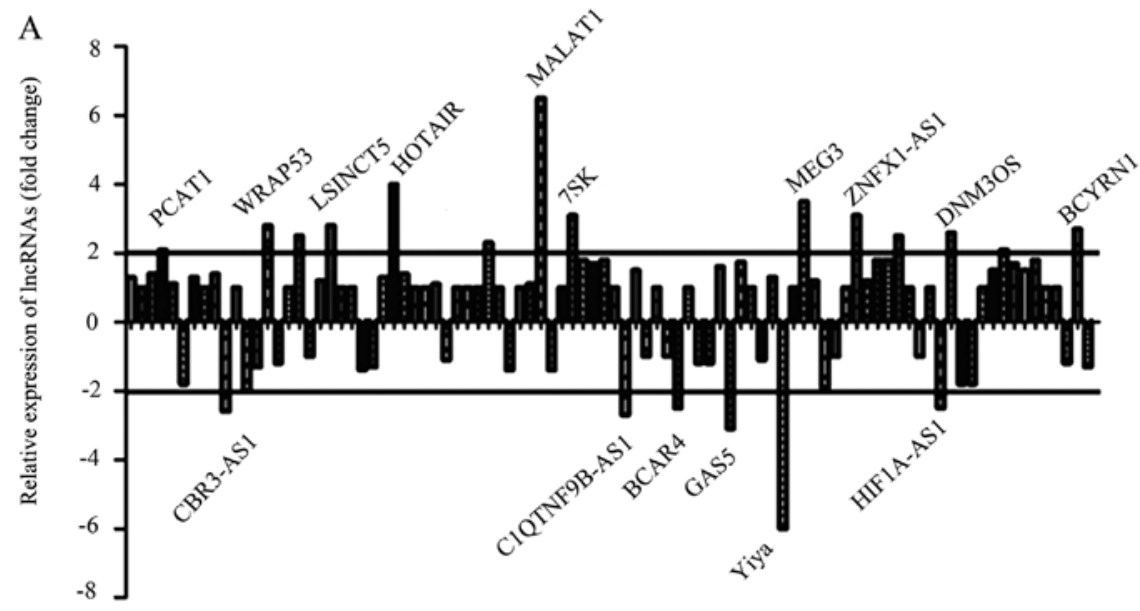

B

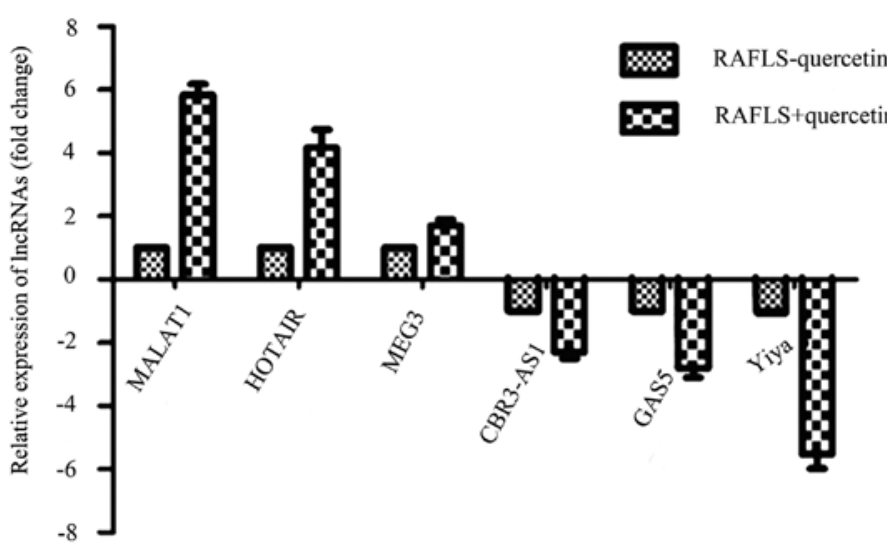

Figure 3. Differentially expressed long non-coding RNAs (lncRNAs) control cells and cells treated with quercetin. (A) Relative levels of lncRNAs which were differentially expressed in the control cells and cells treated with quercetin determined by PCR array. The marked lncRNAs exhibited a difference in expression of $\geq 2$-fold. (B) The relative expression of lncRNAs in the control cells and cells treated with quercetin.

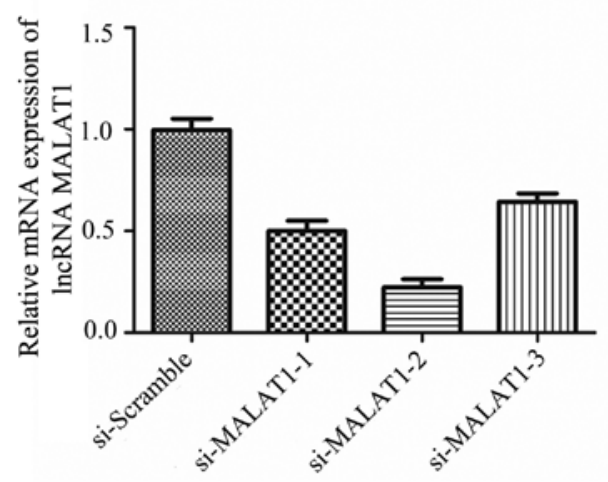

Figure 4. Relative expression of long non-coding RNA (lncRNA) MALAT1 following transfection with siRNA-MALAT1-1, -2, -3 (si-MALAT1-1, -2, -3) or siRNA-Scramble (si-Scramble). MALAT1 was downregulated following transfection with si-MALAT1.

in the levels of P13K and mTOR. These results indicated that the knockdown of MALAT1 led to the activation of the PI3K/AKT pathway. The relative optical density of $\mathrm{p}-\mathrm{P} 13 \mathrm{~K}$,
p-AKT and p-mTOR in the control cells, as well as in the cells transfected with si-Scramble and or si-HOTAIR had been determined previously and the results are shown in Fig. 7. The relative optical density of $\mathrm{p}-\mathrm{P} 13 \mathrm{~K}, \mathrm{p}-\mathrm{AKT}$ and $\mathrm{p}-\mathrm{mTOR}$ in the cells transfected with si-HOTAIR was higher than that of the controls or the cells transfected with si-Scramble.

\section{Discussion}

RA is the most common type of inflammatory arthritis and is characterized by the presence of activated $\mathrm{T}$ lymphocytes, macrophages and synoviocytes. It is a major cause of disability $(31,32)$. RA is also associated with degeneration of cartilage and the erosion of juxta-articular bone, as well as with an increased risk of cardiovascular disease (33,34). FLS are resident mesenchymal cells of the synovial joints which are recognized to play a key role in the pathogenesis of RA (35). It can invade normal human cartilage and bone during the course of RA $(36,37)$. Quercetin, a member of the flavonoid family, is an antioxidant that prevents the oxidation of low-density lipoprotein in vitro by scavenging free oxygen radicals $(21,38)$. It has been shown to induce cell cycle arrest and apoptosis 
A

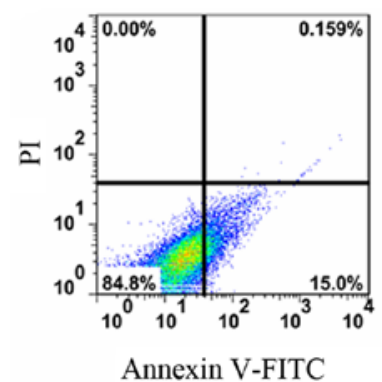

B

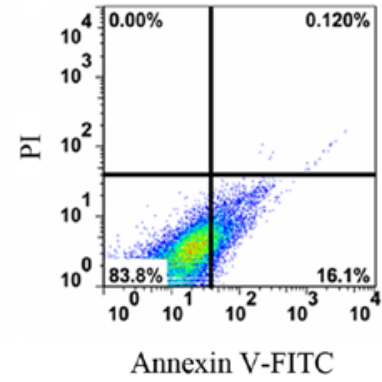

C

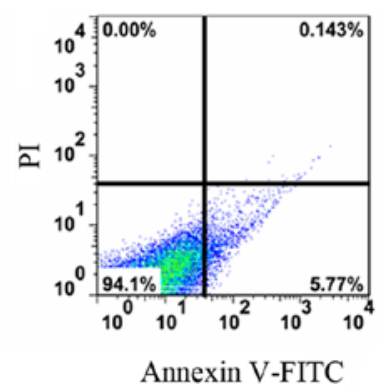

Figure 5. Effect of MALAT1 knockdown on the apoptosis of RAFLS. (A) Control. (B) Cells transfected with siRNA-Scramble (si-Scramble). (C) Cells transfected with siRNA-MALAT1 (si-MALAT1). Upper-left area, dead cells; upper-right area, late-stage apoptotic cells; lower-left area, normal negative cells; lower-right area, early-stage apoptosis cells.

A

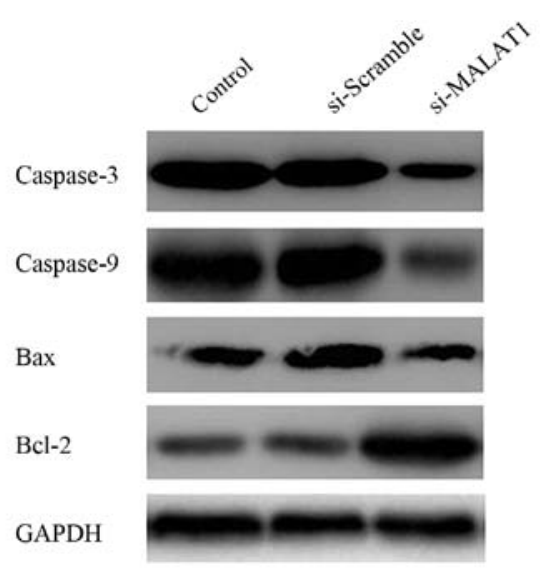

B

PI3K

p-AKT

AKT

p-mTOR

mTOR

GAPDH

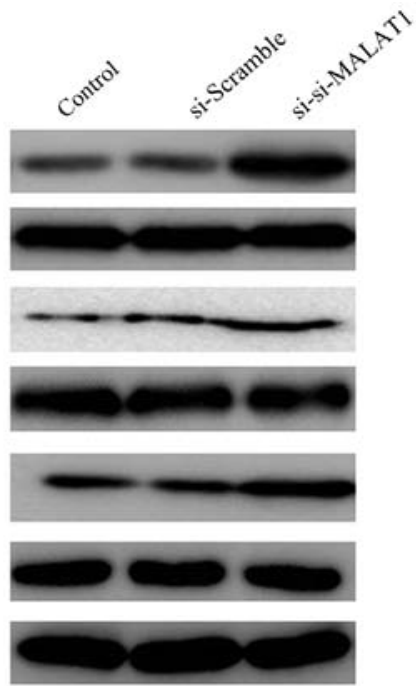

Figure 6. MALAT1 knockdown inhibits the expression of caspase-3 and caspase-9, and activates the PI3K/AKT pathway. (A) Western blot analyses of the caspase-3 and caspase-9, and Bax and Bcl-2 protein expression in RAFLS following transfection with siRNA-Scramble (si-Scramble) or siRNA-MALAT1 (siMALAT1). (B) Western blot analyses of the PI3K/AKT pathway-related proteins in RAFLS.

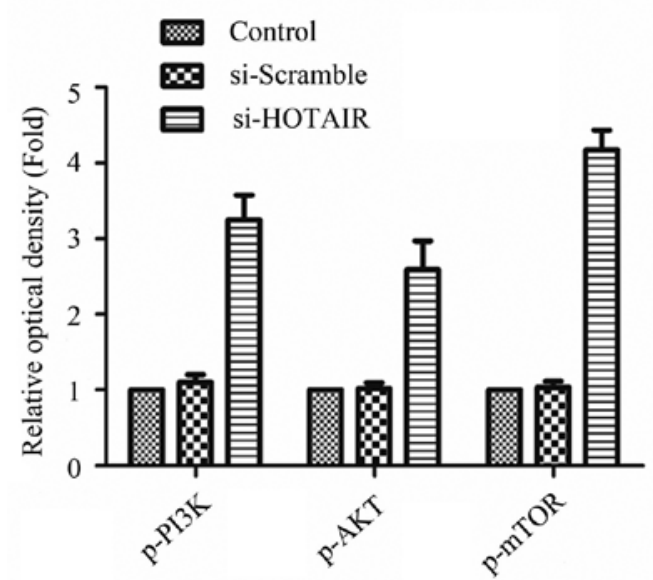

Figure 7. Gray value of p-P13K, p-AKT and p-mTOR in quercetin-treated RAFLS in the control and the group in which with long non-coding RNA (lncRNA) HOTAIR was knocked down by siRNA.

in human breast cancer (22). In human hepatoma cell lines, quercetin has been shown to induce apoptosis by activating caspases, regulating $\mathrm{Bcl}-2$, and inhibiting the activation of the PI3K/AKT and ERK pathways (39). Moreover, it has also been reported to have therapeutic potential for the treatment of RA $(24,40)$. In this study, we aimed to elucidate the molecular mechanisms responsible for the effects of quercetin on RAFLS.

First, the viability of RAFLS following treatment with various concentrations of quercetin for 24, 48 and $72 \mathrm{~h}$ was determined. We found that RAFLS viability markedly decreased following treatment with quercetin in a concentration- and time-dependent manner. The determination of the apoptosis of RAFLS following treatment with $200 \mu \mathrm{M}$ quercetin for 24,48 and $72 \mathrm{~h}$ indicated that the apoptotic rate increased significantly, which was consistent with the results obtained in the study by Sung et al (41). These results indicate that quercetin decreases cell viability and promotes the apoptosis of RAFLS. To elucidate the molecular mechanisms involved, the differentially expressed lncRNAs were screened in the RAFLS treated with quercetin by PCR array and qPCR, and IncRNA MALAT1 was selected for further analysis, as it was the most significantly differentially expressed lncRNA. Transcription generates ncRNAs or lncRNAs, which influence diverse cellular processes, such as cell proliferation, cell cycle progression, apoptosis, or cell growth (42). IncRNAs 
have diverse modes of action and also regulate gene expression $(43,44)$. MALAT1 is an lncRNA that is highly expressed in several types of tumor and its elevated expression is associated with hyper-proliferation $(16,45)$. It plays a pivotal role in colorectal cancer (CRC) metastasis, has been shown to play a key role in the biological processes of cell proliferation, migration and invasion (13). It has been discovered as a marker for lung cancer metastasis, and has been shown to be associated with many RNA binding proteins and is highly conserved throughout evolution (46). Therefore, we inferred that MALAT1 may be a key molecule involved in the mechanisms of action of quercetin. In this study, RAFLS were treated with quercetin after MALAT1 was knocked down and apoptosis was determined. The results revealed that the apoptotic rate of the cells transfected with si-MALAT1 was almost the same as that of the control cells; this indicated that the induction of apoptosis can be prevented following treatjment with quercetin when MALAT1 is knocked down. We thus inferred that MALAT1 is essential for the apoptotic effects of quercetin in RA.

The protein expression of caspase- 3 and $-9, \mathrm{Bcl}-2$ and Bax was determined by western blot analysis. The result revealed that the expression of caspase- 3 and caspase- 9 and Bax decreased, and that of Bcl-2 increased in the cells transfected with si-MALAT1. Caspases are crucial mediators of programmed cell death (apoptosis) (47). Caspase-3 is a member of the cysteine protease family, which plays a key role in apoptotic pathways by cleaving a variety of key cellular proteins (48). It can be activated by various death-inducing signals, including chemotherapeutic agents (49). Caspase- 9 is a member of the caspase family of cysteine proteases that have been implicated in apoptosis and cytokine processing (50). It is one caspase upstream of caspase- 3 and its activation is stimulated by Apaf-1/cytochrome $c$ and inhibited by AKT signals (51). It is critical for cytochrome $c$-dependent apoptosis and normal brain development (52). Bcl-2 family members have been proposed to play a key role in regulating apoptosis (53). The Bcl-2 family can be divided into three classes: the anti-apoptotic Bcl-2, the pro-apoptotic Bax and the BH3-only subfamilies (54). Bax is essential for the death receptor-mediated apoptosis of cancer cells (55). Bcl-2 is an integral membrane protein located mainly on the outer membrane of mitochondria (56). It inhibits most types of apoptotic cell death, implying a common mechanism of lethality (57). The $\mathrm{Bcl}-2 / \mathrm{Bax}$ ratio has been identified to be of clinical relevance in B-cell chronic lymphocytic leukemia (CLL) (58). In this study, the activities of caspase- 3 and caspase- 9 were reduced, and the Bcl-2/Bax ratio was increased following the knockdown of MALAT1. We thus concluded that the apoptosis of RAFLS was inhibited by MALAT1 knockdown.

The relative protein expression of p-P13K, p-AKT, p-mTOR, AKT, P13K and mTOR in the quercetin-treated RAFLS was determined. MALAT1 knockdown significantly enhanced the expression of $\mathrm{p}-\mathrm{P} 13 \mathrm{~K}, \mathrm{p}-\mathrm{AKT}$ and $\mathrm{p}-\mathrm{mTOR}$ and reduced the expression of AKT. However, no detectable changes in P13K and mTOR protein expression were observed. The PI3K/AKT signal transduction cascade has been investigated extensively for its roles in oncogenic transformation (59). PI3K/AKT can regulate the signaling of multiple biological processes such as apoptosis, metabolism, cell proliferation and cell growth (60). Both PI3K and AKT play a role in the prevention of apoptosis. Therefore, we concluded that the knockdown of MALAT1 inhibits cell growth and metastasis by activating the PI3K/ AKT signaling pathway. Moreover, the relative optical density of p-P13K, p-AKT and p-mTOR also increased when HOTAIR was knocked down.

In conclusion, in the present study, we found that MALAT1 expression was significantly upregulated in the quercetintreated RAFLS. The knockdown of MALAT1 inhibited the apoptosis of RAFLS and led to the activation of the PI3K/AKT pathway. Therefore, we concluded that quercetin promotes RAFLS apoptosis by upregulating lncRNA MALAT1.

\section{References}

1. Nanki T, Nagasaka K, Hayashida K, Saita Y and Miyasaka N: Chemokines regulate IL- 6 and IL- 8 production by fibroblast-like synoviocytes from patients with rheumatoid arthritis. J Immunol 167: 5381-5385, 2001

2. Bartok B and Firestein GS: Fibroblast-like synoviocytes: key effector cells in rheumatoid arthritis. Immunol Rev 233: 233-255, 2010.

3. Gabriel SE and Michaud K: Epidemiological studies in incidence, prevalence, mortality, and comorbidity of the rheumatic diseases. Arthritis Res Ther 11: 229, 2009.

4. Kurowska M, Rudnicka W, Kontny E, Janicka I, Chorazy M, Kowalczewski J, Ziółkowska M, Ferrari-Lacraz S, Strom TB and Maśliński W: Fibroblast-like synoviocytes from rheumatoid arthritis patients express functional IL-15 receptor complex: endogenous IL-15 in autocrine fashion enhances cell proliferation and expression of $\mathrm{Bcl}-\mathrm{x}(\mathrm{L})$ and $\mathrm{Bcl}-2$. J Immunol 169: 1760-1767, 2002.

5. Chen S, Yang Y, Feng H, Wang H, Zhao R and Liu H: Baicalein inhibits interleukin-1 $\beta$-induced proliferation of human rheumatoid arthritis fibroblast-like synoviocytes. Inflammation 37: 163-169, 2014.

6. Liu H and Pope RM: The role of apoptosis in rheumatoid arthritis. Curr Opin Pharmacol 3: 317-322, 2003.

7. Pope RM: Apoptosis as a therapeutic tool in rheumatoid arthritis. Nat Rev Immunol 2: 527-535, 2002.

8. Baier A, Meineckel I, Gay S and Pap T: Apoptosis in rheumatoid arthritis. Curr Opin Rheumatol 15: 274-279, 2003.

9. Gloss BS and Dinger ME: The specificity of long noncoding RNA expression. Biochim Biophys Acta 1859: 16-22, 2016.

10. Pedram Fatemi R, Salah-Uddin S, Modarresi F, Khoury N, Wahlestedt $\mathrm{C}$ and Faghihi MA: Screening for small-molecule modulators of long noncoding RNA-protein interactions using AlphaScreen. J Biomol Screen 20: 1132-1141, 2015.

11. Weber DG, Johnen G, Casjens S, Bryk O, Pesch B, Jöckel KH, Kollmeier J and Brüning T: Evaluation of long noncoding RNA MALAT1 as a candidate blood-based biomarker for the diagnosis of non-small cell lung cancer. BMC Res Notes 6: 518, 2013.

12. Jalali S, Bhartiya D, Lalwani MK, Sivasubbu S and Scaria V: Systematic transcriptome wide analysis of 1ncRNA-miRNA interactions. PLoS One 8: e53823, 2013.

13. Xu C, Yang M, Tian J, Wang X and Li Z: MALAT-1: A long non-coding RNA and its important 3 ' end functional motif in colorectal cancer metastasis. Int J Oncol 39: 169-175, 2011.

14. Lai MC, Yang Z, Zhou L, Zhu QQ, Xie HY, Zhang F, Wu LM, Chen LM and Zheng SS: Long non-coding RNA MALAT-1 overexpression predicts tumor recurrence of hepatocellular carcinoma after liver transplantation. Med Oncol 29: 1810-1816, 2012.

15. Ying L, Chen Q, Wang Y, Zhou Z, Huang Y and Qiu F: Upregulated MALAT-1 contributes to bladder cancer cell migration by inducing epithelial-to-mesenchymal transition. Mol Biosyst 8: 2289-2294, 2012.

16. Tripathi V, Shen Z, Chakraborty A, Giri S, Freier SM, Wu X, Zhang Y, Gorospe M, Prasanth SG, Lal A and Prasanth KV: Long noncoding RNA MALAT1 controls cell cycle progression by regulating the expression of oncogenic transcription factor B-MYB. PLoS Genet 9: e1003368, 2013.

17. Dong Y, Liang G, Yuan B, Yang C, Gao R and Zhou X: MALAT1 promotes the proliferation and metastasis of osteosarcoma cells by activating the PI3K/Akt pathway. Tumour Biol 36: 1477-1486, 2015.

18. Bjelogrlić S, Srdić T and Radulović S: Mammalian target of rapamycin is a promising target for novel therapeutic strategy against cancer. J BUON 11: 267-276, 2006. 
19. Gauthier A and Ho M: Role of sorafenib in the treatment of advanced hepatocellular carcinoma: an update. Hepatol Res 43: $147-154,2013$

20. Lamson DW and Brignall MS: Antioxidants and cancer, part 3: quercetin. Altern Med Rev 5: 196-208, 2000

21. Hollman PC, vd Gaag M, Mengelers MJ, van Trijp JM, de Vries JH and Katan MB: Absorption and disposition kinetics of the dietary antioxidant quercetin in man. Free Radic Biol Med 21: 703-707, 1996.

22. Choi JA, Kim JY, Lee JY, Kang CM, Kwon HJ, Yoo YD, Kim TW, Lee YS and Lee SJ: Induction of cell cycle arrest and apoptosis in human breast cancer cells by quercetin. Int J Oncol 19: 837-844, 2001.

23. CoskunO,Kanter M,Korkmaz A and OterS: Quercetin, a flavonoid antioxidant, prevents and protects streptozotocin-induced oxidative stress and $\beta$-cell damage in rat pancreas. Pharmacol Res 51: 117-123, 2005

24. Natarajan V, Krithica N, Madhan B and Sehgal PK: Formulation and evaluation of quercetin polycaprolactone microspheres for the treatment of rheumatoid arthritis. J Pharm Sci 100: 195-205, 2011.

25. Mamani-Matsuda M, Kauss T, Al-Kharrat A, Rambert J, Fawaz F, Thiolat D, Moynet D, Coves S, Malvy D and Mossalayi MD Therapeutic and preventive properties of quercetin in experimental arthritis correlate with decreased macrophage inflammatory mediators. Biochem Pharmacol 72: 1304-1310, 2006

26. De Loof A, Vandersmissen T, Marchal E and Schoofs L: Initiation of metamorphosis and control of ecdysteroid biosynthesis in insects: The interplay of absence of Juvenile hormone, PTTH, and $\mathrm{Ca}(2+)$-homeostasis. Peptides 68: 120-129, 2015.

27. Korb A, Pavenstädt $\mathrm{H}$ and Pap T: Cell death in rheumatoid arthritis. Apoptosis 14: 447-454, 2009.

28. Brunelle JK and Letai A: Control of mitochondrial apoptosis by the Bcl-2 family. J Cell Sci 122: 437-441, 2009.

29. Zhang Y, Sun S, Chen J, Ren P, Hu Y, Cao Z, Sun H and Ding Y: Oxymatrine induces mitochondria dependent apoptosis in human osteosarcoma MNNG/HOS cells through inhibition of PI3K/Akt pathway. Tumour Biol 35: 1619-1625, 2014.

30. Hong SK, Yoon S, Moelling C, Arthan D and Park JI: Noncatalytic function of ERK1/2 can promote Raf/MEK/ERK-mediated growth arrest signaling. J Biol Chem 284: 33006-33018, 2009.

31. Firestein GS: Evolving concepts of rheumatoid arthritis Nature 423: 356-361, 2003 .

32. McInnes IB, al-Mughales J, Field M, Leung BP, Huang FP, Dixon R, Sturrock RD, Wilkinson PC and Liew FY: The role of interleukin-15 in T-cell migration and activation in rheumatoid arthritis. Nat Med 2: 175-182, 1996.

33. Solomon DH, Karlson EW, Rimm EB, Cannuscio CC, Mandl LA, Manson JE, Stampfer MJ and Curhan GC: Cardiovascular morbidity and mortality in women diagnosed with rheumatoid arthritis. Circulation 107: 1303-1307, 2003.

34. Feldmann M and Maini RN: Anti-TNF $\alpha$ therapy of rheumatoid arthritis: what have we learned? Annu Rev Immunol 19: 163-196, 2001.

35. Noss EH and Brenner MB: The role and therapeutic implications of fibroblast-like synoviocytes in inflammation and cartilage erosion in rheumatoid arthritis. Immunol Rev 223: 252-270, 2008.

36. Tolboom TC, van der Helm-Van Mil AH, Nelissen RG, Breedveld FC, Toes RE and Huizinga TW: Invasiveness of fibroblast-like synoviocytes is an individual patient characteristic associated with the rate of joint destruction in patients with rheumatoid arthritis. Arthritis Rheum 52: 1999-2002, 2005.

37. Zhang Q, Wu J, Cao Q, Xiao L, Wang L, He D, Ouyang G Lin J, Shen B, Shi Y, et al: A critical role of Cyr61 in interleukin-17-dependent proliferation of fibroblast-like synoviocytes in rheumatoid arthritis. Arthritis Rheum 60: 3602-3612, 2009.

38. Boots AW, Haenen GR and Bast A: Health effects of quercetin: from antioxidant to nutraceutical. Eur J Pharmacol 585: 325-337, 2008.

39. Granado-Serrano AB, Martín MA, Bravo L, Goya L and Ramos S: Quercetin induces apoptosis via caspase activation, regulation of $\mathrm{Bcl}-2$, and inhibition of PI-3-kinase/Akt and ERK pathways in a human hepatoma cell line (HepG2). J Nutr 136 2715-2721, 2006

40. Sato M, Miyazaki T, Kambe F, Maeda K and Seo H: Quercetin, a bioflavonoid, inhibits the induction of interleukin 8 and monocyte chemoattractant protein-1 expression by tumor necrosis factor-alpha in cultured human synovial cells. J Rheumatol 24: 1680-1684, 1997.
41. Sung MS, Lee EG, Jeon HS, Chae HJ, Park SJ, Lee YC and Yoo WH: Quercetin inhibits IL-1 $\beta$-induced proliferation and production of MMPs, COX-2, and PGE2 by rheumatoid synovial fibroblast. Inflammation 35: 1585-1594, 2012.

42. Wang KC, Yang YW, Liu B, Sanyal A, Corces-Zimmerman R, Chen Y, Lajoie BR, Protacio A, Flynn RA, Gupta RA, et al: A long noncoding RNA maintains active chromatin to coordinate homeotic gene expression. Nature 472: 120-124, 2011.

43. Wapinski $\mathrm{O}$ and Chang HY: Long noncoding RNAs and human disease. Trends Cell Biol 21: 354-361, 2011.

44. Tsai MC, Manor O, Wan Y, Mosammaparast N, Wang JK, Lan F, Shi Y, Segal E and Chang HY: Long noncoding RNA as modular scaffold of histone modification complexes. Science 329: 689-693, 2010

45. Schmidt LH, Spieker T, Koschmieder S, Schäffers S, Humberg J, Jungen D, Bulk E, Hascher A, Wittmer D, Marra A, et al: The long noncoding MALAT-1 RNA indicates a poor prognosis in non-small cell lung cancer and induces migration and tumor growth. J Thorac Oncol 6: 1984-1992, 2011.

46. Eißmann M, Gutschner T, Hämmerle $M$, Günther $S$, Caudron-Herger M, Groß M, Schirmacher P, Rippe K, Braun T, Zörnig M and Diederichs S: Loss of the abundant nuclear noncoding RNA MALAT1 is compatible with life and development. RNA Biol 9: 1076-1087, 2012

47. Porter AG and Jänicke RU: Emerging roles of caspase-3 in apoptosis. Cell Death Differ 6: 99-104, 1999.

48. Devarajan E, Sahin AA, Chen JS, Krishnamurthy RR, Aggarwal N, Brun AM, Sapino A, Zhang F, Sharma D, Yang XH, et al: Down-regulation of caspase 3 in breast cancer: a possible mechanism for chemoresistance. Oncogene 21: 8843-8851, 2002.

49. Du J, Wang X, Miereles C, Bailey JL, Debigare R, Zheng B, Price SR and Mitch WE: Activation of caspase- 3 is an initial step triggering accelerated muscle proteolysis in catabolic conditions. J Clin Invest 113: 115-123, 2004.

50. Kuida K: Caspase-9. Int J Biochem Cell Biol 32: 121-124, 2000.

51. Fujita E, Jinbo A, Matuzaki H, Konishi H, Kikkawa U and Momoi T: Akt phosphorylation site found in human caspase-9 is absent in mouse caspase-9. Biochem Biophys Res Commun 264: $550-555,1999$.

52. Krajewski S, Krajewska M, Ellerby LM, Welsh K, Xie Z, Deveraux QL, Salvesen GS, Bredesen DE, Rosenthal RE, Fiskum G and Reed JC: Release of caspase-9 from mitochondria during neuronal apoptosis and cerebral ischemia. Proc Natl Acad Sci USA 96: 5752-5757, 1999.

53. Lindsten T, Ross AJ, King A, Zong WX, Rathmell JC, Shiels HA, Ulrich E, Waymire KG, Mahar P, Frauwirth K, et al: The combined functions of proapoptotic Bcl-2 family members bak and bax are essential for normal development of multiple tissues. Mol Cell 6: 1389-1399, 2000

54. Oda E, Ohki R, Murasawa H, Nemoto J, Shibue T, Yamashita T, Tokino T, Taniguchi T and Tanaka N: Noxa, a BH3-only member of the Bcl-2 family and candidate mediator of p53-induced apoptosis. Science 288: 1053-1058, 2000.

55. LeBlanc H, Lawrence D, Varfolomeev E, Totpal K, Morlan J, Schow P, Fong S, Schwall R, Sinicropi D and Ashkenazi A: Tumor-cell resistance to death receptor-induced apoptosis through mutational inactivation of the proapoptotic Bcl-2 homolog Bax. Nat Med 8: 274-281, 2002.

56. Fulda S, Meyer E and Debatin K-M: Inhibition of TRAIL-induced apoptosis by Bcl-2 overexpression. Oncogene 21: 2283-2294, 2002.

57. Chipuk JE and Green DR: How do BCL-2 proteins induce mitochondrial outer membrane permeabilization? Trends Cell Biol 18: 157-164, 2008.

58. Saxena A, Viswanathan S, Moshynska O, Tandon P, Sankaran K and Sheridan DP: Mcl-1 and Bcl-2/Bax ratio are associated with treatment response but not with Rai stage in B-cell chronic lymphocytic leukemia. Am J Hematol 75: 22-33, 2004.

59. Chang F, Lee JT, Navolanic PM, Steelman LS, Shelton JG, Blalock WL, Franklin RA and McCubrey JA: Involvement of $\mathrm{PI} 3 \mathrm{~K} / \mathrm{Akt}$ pathway in cell cycle progression, apoptosis, and neoplastic transformation: a target for cancer chemotherapy. Leukemia 17: 590-603, 2003

60. Carnero A, Blanco-Aparicio C, Renner O, Link W and Leal JF: The PTEN/PI3K/AKT signalling pathway in cancer, therapeutic implications. Curr Cancer Drug Targets 8: 187-198, 2008. 